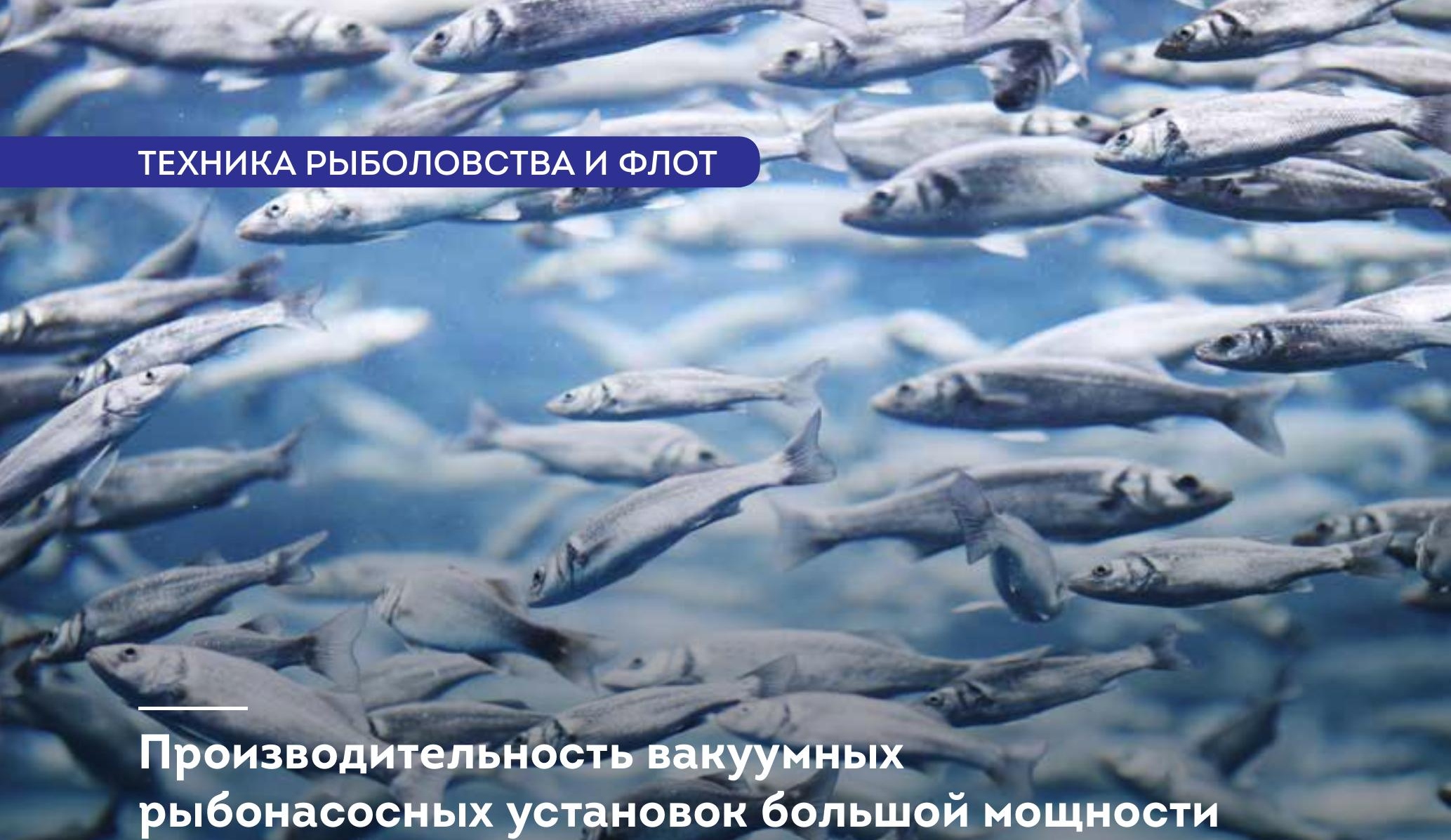
рыбонасосных установок большой мощности

DOI

Д-р техн. наук, профессор В.А. Наумов - Калининградский государственный технический университет (ФГБОУ ВО «КГТУ»), Д-р техн. наук, профессор Н.Л. Великанов - Балтийский федеральный университет им. И.Канта (ФГАОУ ВО «БФУ им. И.Канта"), Студент А.А. Землянов Калининградский государственный технический университет (ФГБОУ ВО «КГТУ»)

@van-old@mail.ru; vladimir.naumov@klgtu.ru; monolit8@yandex.ru; NVelikanov@kantiana.ru; zemlyanov99@gmail.com

Ключевые слова: откачка воздуха, вакуумная рыбонасосная установка, повреждаемость рыбы

Keywords: air pumping out, vacuum fish pumping unit, fish damageability

\title{
PERFORMANCE OF HIGH-POWER VACUUM FISH PUMPING UNITS
}

V. Naumov, Doctor of Sciences, Professor; A. Zemlyanov - Kaliningrad State Technical University; N. Velikanov, Doctor of Sciences, Professor - Kant Baltic Federal University van-old@rambler.ru; zemlyanov99@gmail.com; monolit8@yandex.ru

The article describes the features of modeling the stages of pumping a waterfish mixture using a water-ring vacuum pump. The dynamics of pressure changes in reservoir during pump operation for different time intervals is considered. Solutions of the corresponding differential equations are obtained using the numerical method. The calculated characteristics of the process of water-fish mixture pumping into the receiving container are presented.

Описана динамика изменения производительности работы насоса Samson KS625. Получены решения соответствующих дифференциальных уравнений численным методом. Представлены результаты расчета производительности в процессе перекачивания водорыбной смеси в приемную емкость при различных значениях высоты подъема, продолжительности откачки воздуха, длины трубопровода. Установлено, что для рыбонасосной установки Euskan VS-2000 наибольшего значения производительности можно достигнуть при относительно малых перепадах высот и длинах трубопровода.

Из всех типов устройств для транспорта рыбы вакуумные рыбонасосные установки обеспечивают наименьшую повреждаемость объектов лова. Поэтому в настоящее время они широко применяются, наряду с центробежными рыбонасосами $[1 ; 2]$.

Во время разгрузки промысловых судов необходимы вакуумные рыбонасосные установки с высокой производительностью.
Согласно представляемым данным о технических характеристиках, производительность установок ООО «АгроБалтПроект» по водорыбной смеси может достигать $300 \mathrm{~m}^{3} /$ час (табл. 1), установок Euskan VS - 380 $\mathrm{M}^{3}$ /час (табл. 2).

В [5] была предложена математическая модель полного цикла работы вакуумной рыбонасосной установки, включая 


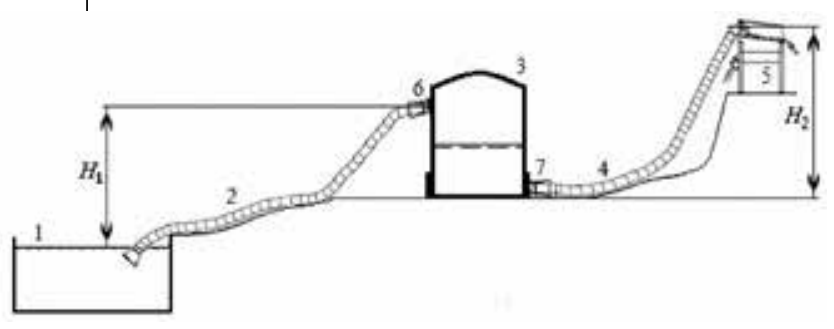

Рисунок 1. Схема Вакуумной рыбонасосной устаноВки: 1 - емкость с водорыбной смесью; 2 - всасывающий трубопровод; 3 - рабочая емкость (резервуар); 4 - нагнетательный трубопровод; 5 - приемная емкость с водоотделителем; 6, 7 - клапаны

Figure 1. Scheme of a vacuum fish pumping unit: 1 - tank with water-fish mixture; 2 - suction pipeline; 3 - reservoir 4 - delivery pipeline; 5 - receiving tank with a water separator; 6, 7 - valves

вторую фазу каждого этапа - движение водорыбной смеси. В данной статье с помощью математической модели [5] выполнен анализ влияния различных факторов на производительность.

Для примера будем использовать в расчетах технические характеристики вакуумной рыбонасосной установки Euskan VS-2000, которая, согласно таблице 1 , имеет производительность $\mathrm{Q}=$ $180 \mathrm{~m}^{3} /$ час, что соответствует $50 \mathrm{дм}^{3} / \mathrm{c}$.

В работе вакуумной рыбонасосной установки применяется циклический принцип, основанный на использовании, с помощью компрессорных машин, поочередно то вакуума, то избыточного давления в рабочей емкости. Из-за такой очередности установка имеет два этапа: всасы-

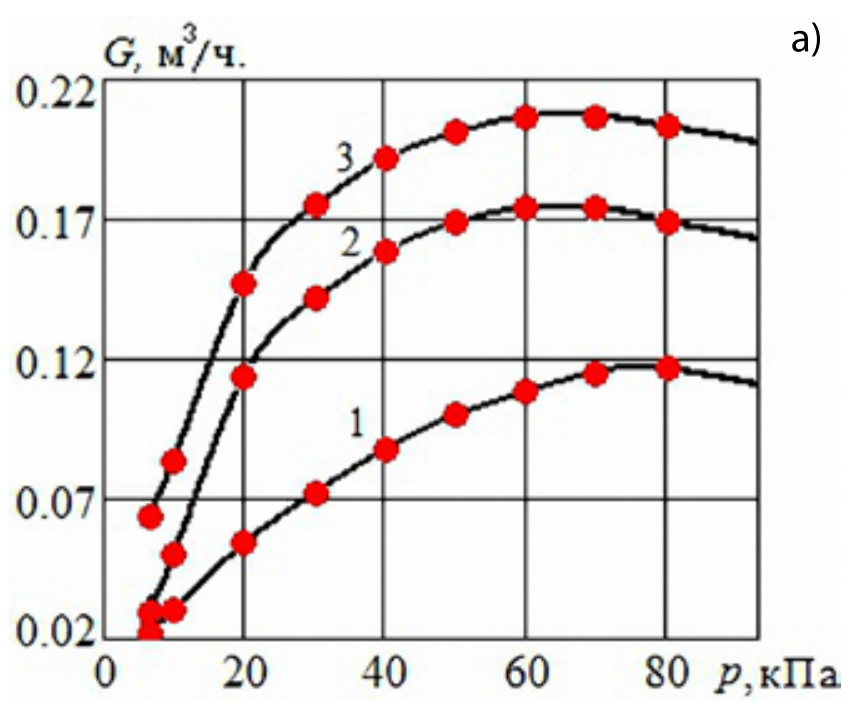

вание и выброс. На этапе всасывания происходит откачивание воздуха из рабочей емкости 3 с помощью вакуумного насоса, водорыбная смесь поступает в резервуар через входной клапан 6 , который расположен в его верхней части. На этапе выброса происходит нагнетание воздуха компрессором в резервуар, водорыбная смесь выбрасывается через клапан 7, расположенный в нижней части резервуара и по нагнетательному трубопроводу 4 подается в приемную емкость 5 (см. puc. 1 [5]).

Вакуумные рыбонасосные установки разных производителей отличаются конструкцией, количеством рабочих емкостей и их объемом, а также схемой управления этапами. Для определенности здесь будем полагать, что имеется резервуар объемом $\mathrm{V}_{0}$. В системе управления сначала происходит откачка воздуха; по ее окончанию открывается клапан 6, и начинается движение жидкости. Так как воздух постоянно соприкасается с водой, можно считать процесс сжатия изотермическим.

Компания Euskan Fish Systems для работы использует водокольцевые компрессорные машины Samson KS625 [6]. Производительность водокольцевой компрессорной машины (расход откачиваемого и нагнетаемого воздуха в рабочую камеру) $\mathrm{G}$ зависит от абсолютного давления в камере и частоты вращения $\mathrm{n}$. Для аппроксимации указанной зависимости использовался метод [7-9] (рис. 2):

$$
\mathrm{G}_{1}=\mathrm{f}_{1}(\mathrm{p}, \mathrm{n}), \mathrm{G}_{2}=\mathrm{f}_{2}(\mathrm{p}, \mathrm{n}),
$$

где индекс 1 относится к работе водокольцевой компрессорной машины в режиме вакуумного насоса, 2 - компрессора.

Далее в расчетах полагаем одинаковыми высоту всасывания и высоту нагнетания рав-

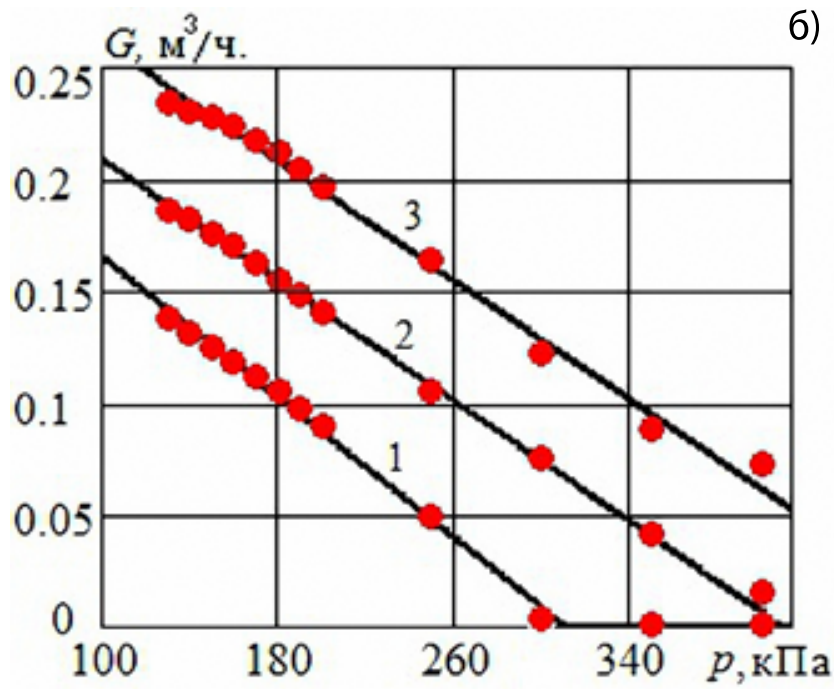

Рисунок 2. Нагрузочные характеристики ВодокольцеВой компрессорной машины Samson KS625: 1 - n=1000 об/мин; 2 - n=1450 об/мин; 3 - n=1750 об/мин).

Точки - экспериментальные данные [6], линии - результаты расчета по (9); a - в режиме вакуумного насоса, b - в режиме компрессора (воздуходувки)

Figure 2. Load characteristics of the Samson KS625 liquid ring compressor machine: $1-n=1000$ rpm; 2 - n = 1450 rpm; $3-n=1750 \mathrm{rpm}$. Points represent experimental data [6], lines - calculation results according to (9); a - in vacuum pump mode, b - in compressor (blower) mode 
Таблица 1. Технические характеристики вакуумной рыбонасосной установки AB510R [3] /

Table 1. Technical characteristics of the AV510R vacuum fish pumping unit [3]

\begin{tabular}{|c|c|c|c|c|}
\hline Объем бака, л & 500 & 1000 & 1500 & 2000 \\
\hline Диаметр входа/выхода, мм & $150(200)$ & $200(250)$ & 250 & 300 \\
\hline Производительность, м³/час (рыба+вода) & 75 & 110 & 175 & 210 \\
\hline Средний вес рыбы, кг & до 4 & до 6 & 6 & 8 \\
\hline Мощность ВКМ, кВт & 11 & 15 & 22 & 37 \\
\hline
\end{tabular}

У всех моделей указана максимальная общая высота подъема - 9 м (в том числе всасывания - до 5 м, нагнетания - до 4 м).

Таблица 2. Технические характеристики вакуумной рыбонасосной установки Euskan VS [4] /

Table 2. Technical characteristics of the Euskan VS vacuum fish pumping unit [4]

\begin{tabular}{|c|c|c|c|c|c|c|}
\hline Объем бака, л & 500 & 1000 & 1500 & 2000 & 3000 & 4500 \\
\hline Диаметр входа/выхода, мм & 200 & 250 & 250 & 300 & 300 & 350 \\
\hline Производительность, м3/час & 60 & 95 & 160 & 180 & 260 & 380 \\
\hline Модель ВКМ Samson & KE225 & KL350 & KS510 & KS625 & KS910 & KM2200 \\
\hline Мощность ВКМ, кВт & 11 & 15 & 22 & 37 & 55 & 75 \\
\hline
\end{tabular}

ными $\mathrm{H}_{1}=\mathrm{H}_{2}=\mathrm{H}_{0}$, длину всасывающего и нагнетательного трубопровода также равными $\mathrm{L}_{1}=\mathrm{L}_{2}=\mathrm{L} ; \mathrm{V}_{0}=2 \mathrm{~m}^{3} ; \theta=0,2 ; \mathrm{k}=0,03 ; \mathrm{d}=0,3 \mathrm{~m}$.

Как было показано в [5], течение жидкости, как во всасывающем рукаве, так и в нагнетательном трубопроводе, является нестационарным процессом, из-за изменения разности давлений. По результатам расчета, представленным на рисунке 3 , видно, что при небольшой высоте подъема $\left(\mathrm{H}_{0}=1,2 \mathrm{~m}\right)$ мгновенный расход водорыбной смеси может превысить $600 \mathrm{gm}^{3} / \mathrm{c}$. С увеличением высоты подъема он уменьшается, но и при $\mathrm{H}_{0}=7,5$ м все еще достигает 200 дм $^{3} /$ с. Однако производительность вакуумной рыбонасосной установки за цикл значительно ниже.

Производительность вакуумной рыбонасосной установки рассчитывается как частное

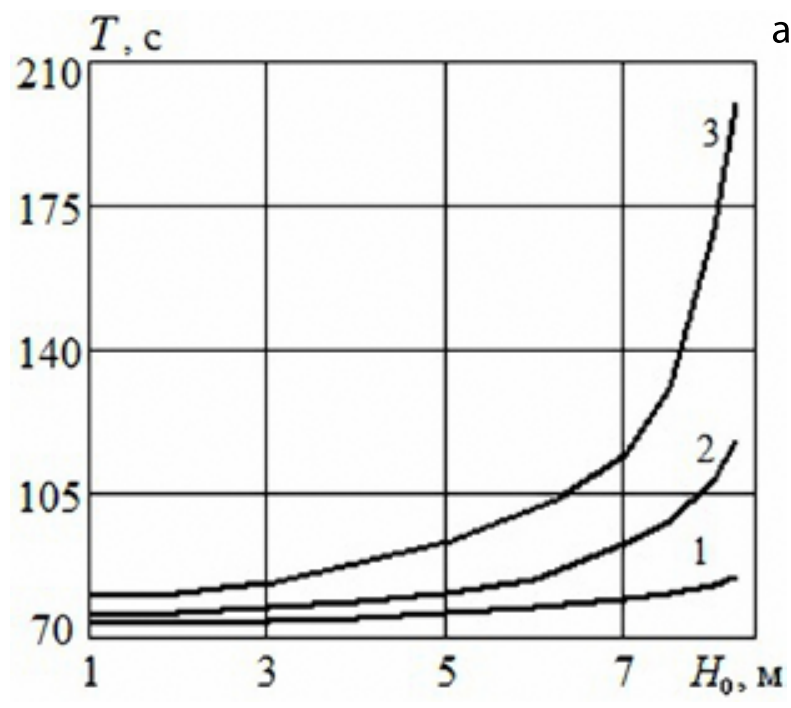

a)

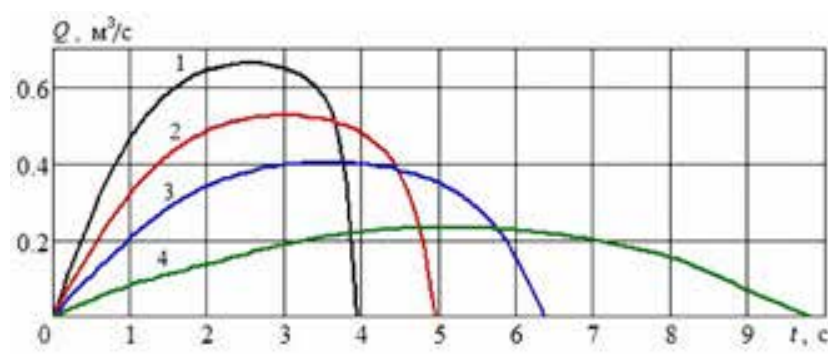

Рисунок 3. Изменение расхода во всасывающем рукаве при $\mathrm{n}=1450$ об/ мин, $T_{11}=60 \mathrm{c}, \mathrm{L}=10 \mathrm{M}: 1-\mathrm{H}_{0}=1,2 \mathrm{M}$; $2-\mathrm{H}_{0}=4 \mathrm{M} ; 3-\mathrm{H}_{0}=6 \mathrm{M} ; 4-\mathrm{H}_{0}=7,5 \mathrm{M}$

Figure 3. Change in the flow rate in the suction hose at $\mathrm{n}=1450 \mathrm{rpm}, \mathrm{T}_{11}=60 \mathrm{~s}, \mathrm{~L}=10 \mathrm{~m}: 1-\mathrm{H}_{0}=1.2 \mathrm{~m} ; 2-\mathrm{H}_{0}=4 \mathrm{~m}$; $3-\mathrm{H}_{0}=6 \mathrm{~m} ; 4-\mathrm{H}_{0}=7.5 \mathrm{~m}$

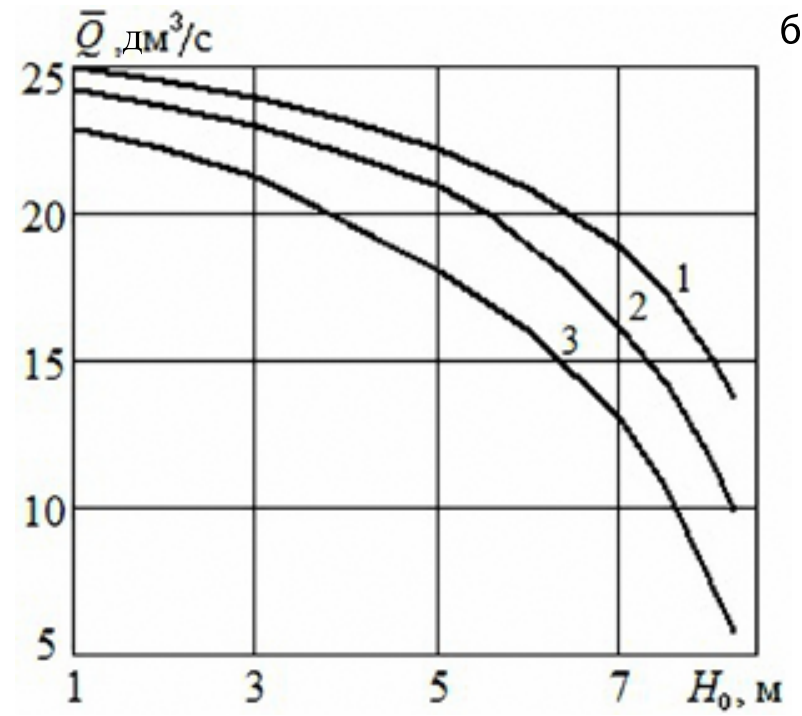

Рисунок 4. Влияние высоты подъема на продолжительность цикла (а) и производительность вакуумной рыбонасосной установки (b) при $\mathrm{n}=1450$ об/мин и различной длине трубопровода: 1 - L=10 м; 2 - L=50 м; 3 - L=100 м

Figure 4. Influence of the lifting height on the cycle time (a) and the productivity of the vacuum fish pumping unit (b) at $\mathrm{n}=1450 \mathrm{rpm}$ and different pipeline lengths: $1-\mathrm{L}=10 \mathrm{~m} ; 2-\mathrm{L}=50 \mathrm{~m} ; 3-\mathrm{L}=100 \mathrm{~m}$ 
Таблица 3. Результаты расчета при $\mathrm{n}=1450$ об/мин, $\mathrm{T}_{11}=60 \mathrm{c}, \mathrm{L}=10 \mathrm{~m} /$

Table 3. Calculation results at $\mathrm{n}=1450 \mathrm{rpm}, \mathrm{T}_{11}=60 \mathrm{~s}, \mathrm{~L}=10 \mathrm{~m}$

\begin{tabular}{cccc}
\hline $\mathbf{H}_{1}, \mathbf{M}$ & $\mathbf{V}_{1}, \mathrm{M}^{3}$ & $\mathbf{T}, \mathbf{c}$ & $\overline{\mathbf{Q}}, \mathbf{A M}^{3} / \mathbf{c}$ \\
\hline 1,2 & 1,820 & 73,2 & 24,9 \\
4,0 & 1,738 & 75,0 & 23,2 \\
6,0 & 1,613 & 77,3 & 20,9 \\
7,5 & 1,396 & 80,9 & 17,3 \\
7,5 & 1,396 & 80,9 & 17,3 \\
\hline
\end{tabular}
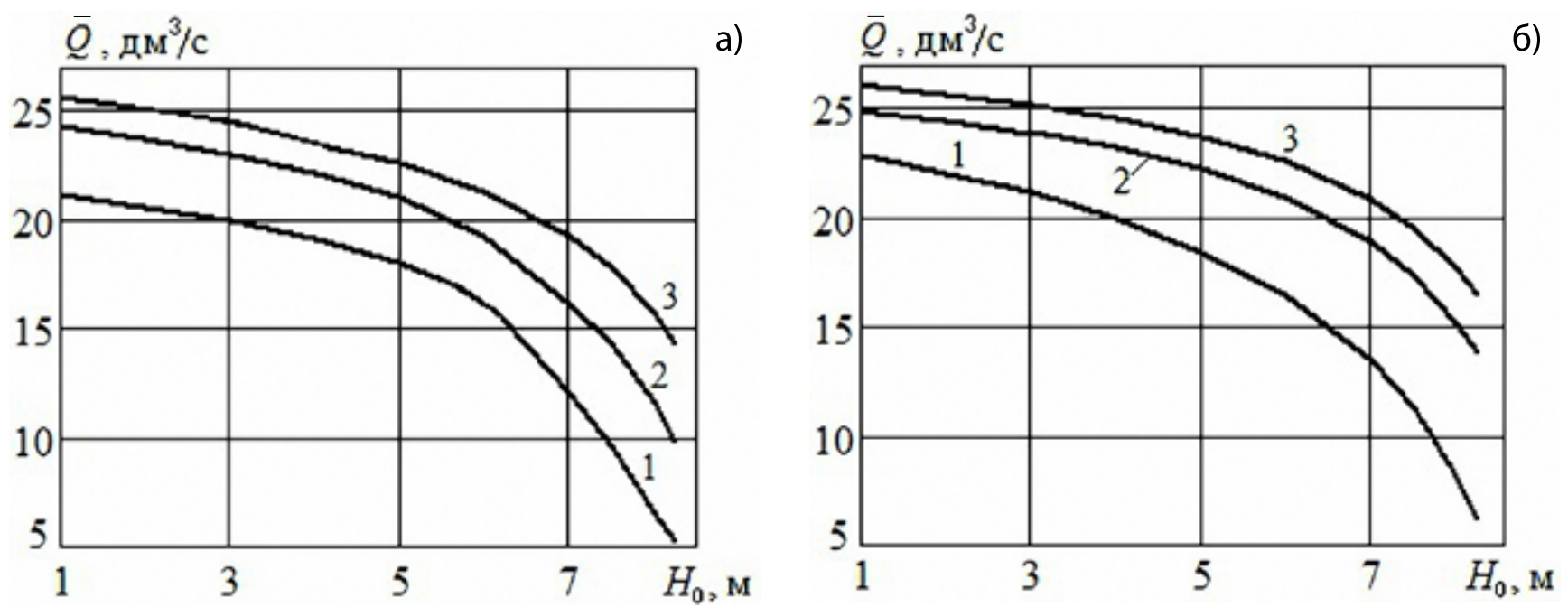

Рисунок 5. Влияние высоты подъема на производительность вакуумной рыбонасосной установки при, $T_{11}=60$ c, a - при L=50 м, $\mathrm{n}=1450$ об/мин и разных диаметрах рукава: 1 - d=0,2 м; 2 - d=0,3 м; 3 - d=0,4 м; b - при L=10 м, d=0,3 м и разной частоте вращения: 1 - n = 1000 об/мин; $2-\mathrm{n}=1450$ об/мин; $3-\mathrm{n}=1750$ об/мин.

Figure 5. Influence of the lifting height on the performance of the vacuum fish pumping unit at, $T_{11}=60 \mathrm{~s}, \mathrm{a}-$ at $\mathrm{L}=50 \mathrm{~m}, \mathrm{n}=$ $1450 \mathrm{rpm}$ and different diameters of the sleeve: $1-d=0.2 \mathrm{~m} ; 2-d=0.3 \mathrm{~m} ; 3-d=0.4 \mathrm{~m} ; \mathrm{b}-\mathrm{at} L=10 \mathrm{~m}, d=0.3 \mathrm{~m}$ and different rotation speeds: $1-n=1000$ rpm; $2-n=1450$ rpm; $3-n=1750$ rpm.
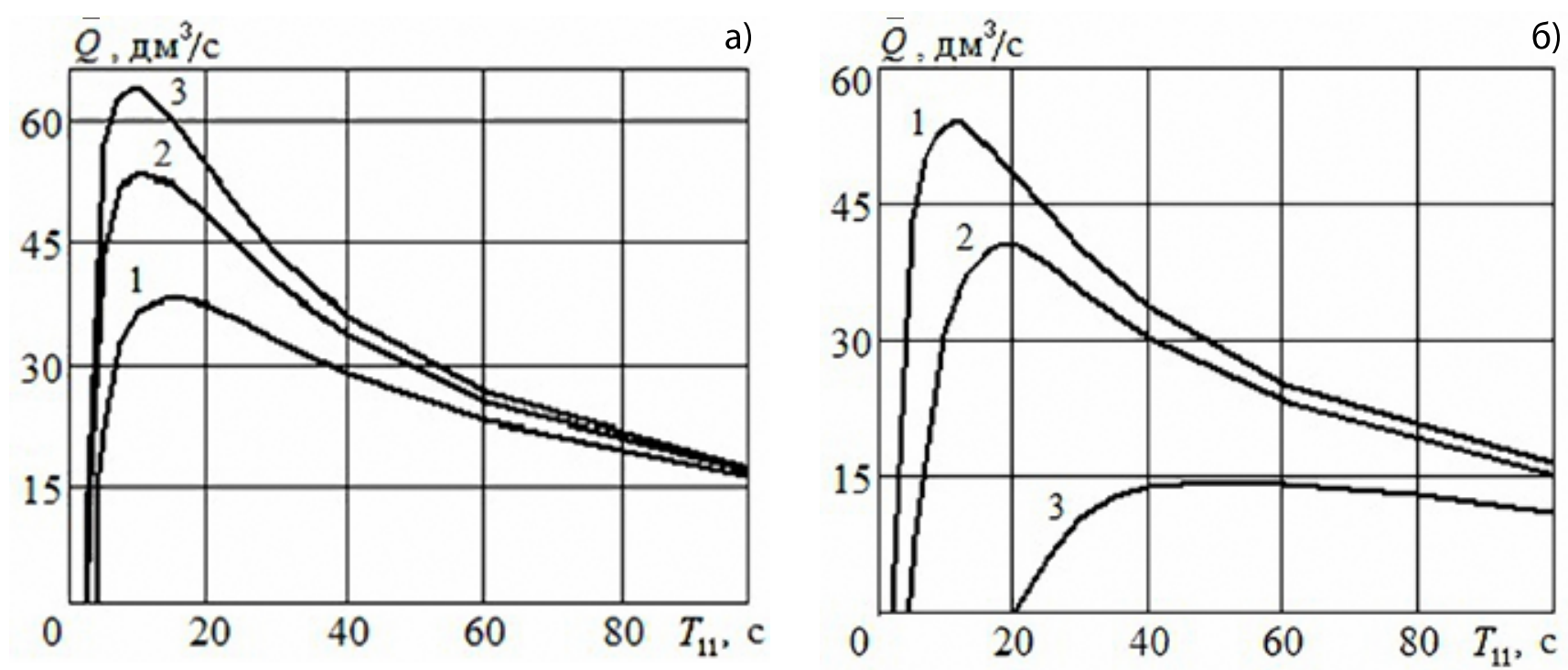

Рисунок 6. Влияние продолжительности откачки воздуха на производительность вакуумной рыбонасосной установки, а - при L= 3 м; $\mathrm{H}_{0}=1,2$ м и разной частоте вращения: 1 - $\mathrm{n}=1000$ об/ мин, $2-\mathrm{n}=1450$ об/мин, $3-\mathrm{n}=1750$ об/мин;

b - при $n=1000$ об/мин: $1-L=3$ м; $H_{0}=1,2$ м; $2-L=20$ м; $H_{0}=3$ м; $3-L=20$ м; $H_{0}=8$ м

Figure 6. Influence of air pumping duration on the productivity of the vacuum fish pumping unit a - at $L=3 \mathrm{~m} ; \mathrm{H}_{0}=1.2 \mathrm{~m}$ and different speeds: $1-n=1000 \mathrm{rpm}, 2-n=1450 \mathrm{rpm}, 3-\mathrm{n}=1750 \mathrm{rpm} ; \mathrm{b}-$ at $n=1000 \mathrm{rpm}: 1-\mathrm{L}=3 \mathrm{~m} ; \mathrm{H}_{0}=1.2 \mathrm{~m} ; 2-\mathrm{L}=20 \mathrm{~m}$; $\mathrm{H}_{0}=3 \mathrm{~m} ; 3-\mathrm{L}=20 \mathrm{~m} ; \mathrm{H}_{0}=8 \mathrm{~m}$ 
от деления объема жидкости $\mathrm{V}_{1}$, перекачанной за один цикл, на полное время цикла Т:

$$
\overline{\mathrm{Q}}=\mathrm{V}_{1} / \mathrm{T}, \mathrm{T}=\mathrm{T}_{11}+\mathrm{T}_{12}+\mathrm{T}_{21}+\mathrm{T}_{22},
$$

где $\mathrm{T}_{11}$ - продолжительность первой фазы первого этапа (откачка воздуха из рабочей камеры), $\mathrm{T}_{12}$ - продолжительность второй фазы первого этапа (закачка водорыбной смеси в рабочую камеру), $\mathrm{T}_{21}$ - продолжительность первой фазы второго этапа (нагнетание воздуха в рабочую камеpy), $\mathrm{T}_{22}$ - продолжительность второй фазы второго этапа (вытеснение водорыбной смеси из рабочей камеры).

При заданном объеме рабочей камеры $\mathrm{V}_{0}$, объем жидкости, перекачиваемой за один цикл, определяется давлением в рабочей камере в конце первой фазы первого этапа $\mathrm{p}_{0}$ и высотой всасывания $\mathrm{H}_{1}$. Абсолютное давление в камере $\mathrm{p}_{0}=8,06$ кПа при $\mathrm{n}=1450$ об/мин, $\mathrm{T}_{11}=60$ с. Результаты расчета среднего расхода, представленные в таблице 3 , заметно меньше производительности, указанной в техническом паспорте 50 дм $^{3} / \mathrm{c}$.

Исследуем влияние длины трубопровода при условиях, указанных в таблице 3 . Величина $\mathrm{V}_{1}$ не зависит от длины рукава, остается такой же, как в таблице. 3. С увеличением протяженности трубопровода растут гидравлические потери, снижается скорость движения водорыбной смеси. Время цикла работы вакуумной рыбонасосной установки возрастает (рuc. 4a), в результате - производительность будет тем меньше, чем больше длина трубопровода (рuc. 4b).

BKM Samson KS625 может работать при трех значениях частоты вращения. Наибольшая производительность (но и наибольшие энергозатраты) будет при $\mathrm{n}=1750$ об/мин, так как снижается время цикла (рuс. 5a). Увеличение диаметра трубопровода приводит к снижению гидравлических потерь и росту производительности (рuc. $5 b)$.

Количество водорыбной смеси, перекачиваемой за один цикл, зависит от давления $\mathrm{p}_{0}$ в камере в конце первой фазы первого этапа. Величину этого давления можно регулировать, устанавливая продолжительность откачивания воздуха из рабочей камеры с помощью водокольцевой компрессорной машины $\mathrm{T}_{11}$. На рисунке 6 показано, как влияет $\mathrm{T}_{11}$ на производительность.

По рисунку ба видно, что только при малых значениях $\mathrm{H}_{0}$ и L, устанавливая $\mathrm{T}_{11}$ около $10 \mathrm{c}$, можно добиться производительности выше 50 дм $^{3} / \mathrm{c}$ (n = 1450 об/мин) или даже выше 50 дм $^{3} / \mathrm{c}$ (n = 1750 об/мин). Однако так уменьшать величину $\mathrm{T}_{11}$ нельзя при немалых значениях высоты подъема и длины трубопровода. На рисунке $6 \mathrm{~b}$ линия 3 показывает, что при $\mathrm{L}=20 \mathrm{M} ; \mathrm{H}_{0}=8$ м необходимо установить $\mathrm{T}_{11}>20$ с (лучше 40-60 с), иначе водокольцевая компрессорная машина не успеет откачать воздух в рабочей камере до необходимого давления, и установка просто не будет работать.

\section{ЛИТЕРАТУРА И ИСТОЧНИКИ}

1. Кудакаев В.В. Автоматизированные гидравлические системы транспортировки рыбы из орудий лова рыбонасосами / В.В. Ку- дакаев, Т.П. Карпелев, А.Н. Бойцов // Известия ТИНРО. 2016. Т. 186. - С. 207-213.

1. Kudakaev V.V. Avtomatizirovannye gidravlicheskie sistemy transportirovki ryby iz orudij lova rybonasosami / V.V. Kudakaev, T.P. Karpelev, A.N. Bojcov // Izvestiya TINRO. 2016. V. 186. - Pp. 207213.

2. Великанов Н.Л. Компрессорные машины вакуумных рыбонасосов / Н.Л. Великанов, В.А Наумов // Рыбное хозяйство. 2018. № 6. - С. 78-81.

2. Velikanov N.L. Kompressornye mashiny vakuumnyh rybonasosov / N.L. Velikanov, V.A Naumov // Rybnoe hozyajstvo. 2018. Issue 6. - Pp. 78-81.

3. ООО «АгроБалтПроект» [Электронный ресурс]. - URL: http:// www.agro-balt.ru/ (дата обращения: 02.01.2020).

3. OOO «AgroBaltProekt» [Web resource]. http://www.agro-balt.ru/ (visited 02.01.2020).

4. Euskan Fish Systems [Электронный ресурc]. - URL: http://www. euskan.com/ (дата обращения: 02.01.2020).

4. Euskan Fish Systems [Web resource]. http://www.euskan.com/ (visited 02.01.2020).

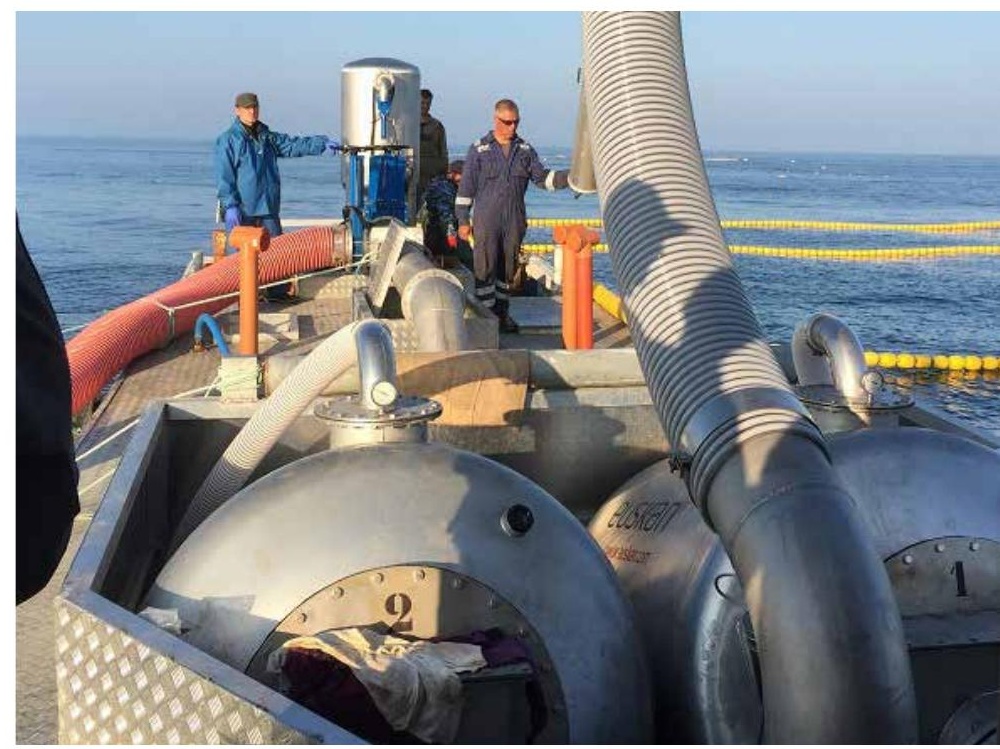

5. Наумов В.А. Этапы работы вакуумной рыбонасосной установки / В.А. Наумов, Н.Л. Великанов // Рыбное хозяйство. 2020. № 2. - C. 108-112.

5. Naumov V.A. Etapy raboty vakuumnoj rybonasosnoj ustanovki / V.A. Naumov, N.L. Velikanov // Rybnoe hozyajstvo. 2020. Issue 2. Pp. 108-112.

6. Samson Liquid Ring Vacuum Pumps. Catalogs of the equipment [Электронный ресурс]. - URL: www.samson-pumps.com/ (дата обращения: 02.01.2020).

6. Samson Liquid Ring Vacuum Pumps. Catalogs of the equipment [Web resource]. www.samson-pumps.com/ (visited 02.01.2020).

7. Naumov V.A. Simulation of operational characteristics of the water-ring vacuum pumps / V.A. Naumov, N.L. Velikanov // IOP Conf. Series: Materials Science and Engineering. - 2019. - 537, 032029.

7. Naumov V.A. Simulation of operational characteristics of the waterring vacuum pumps / V.A. Naumov, N.L. Velikanov // IOP Conf. Series: Materials Science and Engineering. - 2019. - 537, 032029.

8. Великанов Н.Л. Моделирование характеристик водокольцевых вакуумных насосов / Н.Л. Великанов, В.А. Наумов / / Известия вузов. Машиностроение. 2019. № 10. - С. 70-77.

8. Velikanov N.L. Modelirovanie harakteristik vodokol'cevyh vakuumnyh nasosov / N.L. Velikanov, V.A. Naumov // Izvestiya vuzov. Mashinostroenie. 2019. Issue 10. - Pp. 70-77.

9. Великанов Н.Л. Динамические характеристики вакуумных насосов и компрессоров рыбонасосных установок / Н.Л. Великанов, В.А. Наумов // Рыбное хозяйство. 2019. № 1. - С. 79-83.

9. Velikanov N.L. Dinamicheskie harakteristiki vakuumnyh nasosov i kompressorov rybonasosnyh ustanovok / N.L. Velikanov, V.A. Naumov // Rybnoe hozyajstvo. 2019. Issue 1. - Pp. 79-83. 